\title{
É POSSÍVEL FAZER UMA ANÁLISE COMPORTAMENTAL DA INTELIGÊNCIA?
}

\section{IS A BEHAVIORAL ANALYSIS OF INTELLIGENCE POSSIBLE?}

\author{
LAURA Z. RABELO \\ JULIO C. DE ROSE \\ UNIVERSIDADE FEDERAL DE SÃo CARLOS, BRASIL E \\ InStituto NACIONAL de CIÊNCIA E TECNOLOGIA SOBRE COMPORTAMENTO, COGNIÇÃo E ENSINO, BRASIL

\section{RESUMO}

O estudo da inteligência iniciou-se no século XIX com a investigação da origem das diferenças individuais. Desde então, variados testes, teorias e modelos de inteligência foram criados. Durante várias décadas, a utilidade do conceito "inteligência" foi questionada por analistas do comportamento, uma vez que eles buscam identificar as variáveis ambientais que controlam o comportamento. O objetivo deste artigo é revisar a literatura que aborda a inteligência por uma perspectiva comportamental, baseando-se principalmente nas Teorias de Equivalência de Estímulos e das Molduras Relacionais. Algumas críticas da área ao conceito de inteligência serão apresentadas, bem como explorar-se-á uma possibilidade comportamental de análise desse construto. As evidências empíricas que apontam uma conexão entre repertórios de responder relacional derivado e o funcionamento intelectual serão discutidas. O diálogo entre a Análise do Comportamento e a Psicometria permite que as contribuições das duas se complementem para o planejamento de intervenções efetivas, o que é visto como o objetivo maior de ambas as áreas.

Palavras-chave: inteligência, molduras relacionais, responder relacional derivado, equivalência de estímulos, psicometria, intervenção

Intelligence began being studied in the 19th Century when the source of individual differences was investigated. Since then, numerous intelligence tests, theories and models were developed. For many decades, behavior analysts questioned the utility of "intelligence" concept, as they consider that variables that exert control on behavior are to be found in the environment. This article reviews the literature that deals with intelligence from a behavioral perspective, leaning mainly on the Stimulus Equivalence and Relational Frame Theories to do so. Some of the criticism made to the concept of intelligence will be presented, as well as a possibility of a behavioral analysis of it. Additionaly, empirical evidences that support the connection between derived relational responding repertoires and intellectual functioning will be discussed. The dialogue between Behavior Analysis and Psychometrics enables their contributions to complement each other for planning effective interventions, which is the ultimate objective of both areas. intervention

Keywords: intelligence, relational frames, derived relational responding, stimulus equivalence, psychometrics,

Os autores fazem parte do Instituto Nacional de Ciência e Tecnologia sobre Comportamento, Cognição e Ensino (INCTECCE) apoiado pelo Ministério da Ciência, Tecnologia e Inovação (MCTI - edital 15/2008), por meio do CNPq (Processo \# 573972/2008-7) e da FAPESP (Processo \#2008/57705-8). A primeira autora agradece o apoio da FAPESP (Processo \#2012/24018-3) e da CAPES (Processo \#99999.010581/2014-06) por possibilitarem o desenvolvimento deste trabalho. Os autores também agradecem os comentários e sugestões de um revisor anônimo. Endereço para correspondência: Departamento de Psicologia da Universidade Federal de São Carlos (Laboratório de Estudos do Comportamento Humano - LECH). Rodovia Washington Luís, km 235, s/n - Jardim Guanabara, São Carlos - SP, 13565905. Email dos autores: laurazrabelo@gmail.com e julioderose@ gmail.com. 
O estudo da inteligência e o estabelecimento da Psicometria como um campo de investigação ocorreram de maneira entrelaçada. A partir do século XIX, a fonte das diferenças individuais tornou-se motivo de interesse científico. Francis Galton (1822-1911) interessava-se em identificar indivíduos que poderiam produzir uma prole talentosa e, para isso, criou uma série de avaliações que mediam o tempo de reação a estímulos e a acuidade dos sentidos das pessoas. Seu primo, Charles Darwin, havia escrito o livro A origem das espécies, que influenciou profundamente as suas investigações. Nesse contexto, nascia a área que hoje conhecemos como Psicometria (Schelini, 2006; Wasserman \& Tulsky, 2005).

No entanto, foi apenas no início do século XX que Alfred Binet (1857-1911) mudou o foco das investigações de medidas físicas para o estudo das "faculdades mentais". Binet trabalhava inicialmente com a craniometria, que tinha o pressuposto de que a medida encefálica de pessoas mais "inteligentes" era maior do que a das menos inteligentes. Com o passar do tempo, ele percebeu que os pressupostos da craniometria não se sustentavam e passou a investigar processos "mentais", quando o ministro francês da educação pública o contatou para que ele criasse uma avaliação com o intuito de identificar alunos que precisariam de educação especial (Gould, 1991).

Em 1905, ele produziu sua primeira escala. Binet dizia ter criado uma escala que buscava avaliar a "inteligência natural" das crianças, separando-a do que havia sido aprendido na escola. Trabalhou em sua escala com Théodore Simon (1872-1961) e, por isso, o nome de Escala Binet-Simon. Os testes propostos por eles envolviam habilidades cotidianas e eram muito numerosos. O termo "inteligência" foi usado em um dos livros de Binet para descrever a soma total dos processos mentais (Gould, 1991). Etimologicamente, o termo "inteligência" vem do latim intelligere e significa compreender ou entender algo (Wasserman \& Tulsky, 2005). A versão original da escala, e também a segunda versão, introduziram parâmetros que são usados até hoje: a ordenação dos testes por níveis de complexidade (1905) e a versão preliminar do quociente intelectual (QI), que era calculado por meio da subtração da idade mental (obtida por meio das tarefas que as crianças acertaram), da sua idade cronológica (1908). Contudo, foi W. Stern, em 1912, que sugeriu que o QI fosse calculado da forma como fazemos hoje, isto é, dividindo a idade mental pela cronológica e multiplicando por 100 (Gould, 1991).

Lewis Terman (1877-1956) fez a adaptação da Escala Binet-Simon para a população dos Estados Unidos, nomeando-a "Escala Stanford-Binet". No período da Primeira Guerra Mundial, ele criou baterias de avaliação para os soldados do exército e adaptou-as para a aplicação em grupo. David Wechsler (1896-1971) foi enormemente influenciado por essas baterias de avaliação do exército. Ele selecionou e organizou testes extraídos delas para criar sua própria escala em 1939, a Escala Wechsler-Bellevue. Em meados dos anos 50 a popularidade da Escala Wechsler-Bellevue já havia superado a Stanford-Binet e até hoje suas sucessoras (WISC para crianças e WAIS para adultos) continuam sendo muito usadas ao redor do mundo. Sua popularidade pode ser atribuída a diversos fatores, dentre eles, a carência de outros testes para adultos, a integração dos testes verbais e de desempenho dentro de uma bateria, a ênfase no rigor psicométrico, uma amostra de normatização que atendia aos mais modernos critérios de seleção para a época, e a conformidade com os testes que já eram comumente usados (Wasserman \& Tulsky, 2005).

O processo atual de construção e validação de testes psicológicos é longo e bastante complexo. Inicialmente, o construto que embasará o teste deve ser extensivamente pesquisado na literatura para que sua descrição e delimitação sejam adequadas e, também, para garantir que haja a necessidade de um novo teste sobre o assunto. A partir disso, um conjunto de itens que abranjam todos os aspectos relevantes do construto é criado. Após a criação dos itens, eles são aplicados em uma amostra de cerca de 300 pessoas, que seja representativa da heterogeneidade encontrada na população alvo da escala. O próximo passo relaciona-se com as análises de consistência interna (grau em que os itens que formam a escala são relacionados entre si). Finalmente, são feitas as análises fatoriais exploratórias e confirmatórias. A primeira identifica quantos fatores há na escala e agrupa os itens relacionados com cada fator. A análise confirmatória, por sua vez, compara os dados obtidos pela aplicação do teste com a teoria que o embasou (para mais detalhes, ver Clark \& Watson, 1995).

David Wechsler fez outra contribuição à área, que foi a definição de inteligência. Em 1921, 17 pesquisadores foram convidados pelo Journal of Educational Psychology a definir inteligência. Diante da pluralidade de definições e da falta de consenso, a definição mais utilizada é aquela dada por Wechsler. Para ele, a inteligência é uma capacidade geral do indivíduo de lidar com seu ambiente de forma eficaz, de agir com propósito, e de pensar racionalmente (Wasserman \& Tulsky, 2005).

Os dados de avaliações acadêmicas em larga escala como o SAT (Student Achievement Test) nos Estados Unidos, um teste equivalente ao ENEM (Exame Nacional do Ensino Médio) para o caso do Brasil, têm uma forte correlação com os escores de testes de inteligência (Schroeders, Wilhelm, \& Bucholtz, 2010). De fato, isso indica que os testes de inteligência avaliam habilidades relevantes para o contexto acadêmico. No entanto, os resultados desses testes frequentemente são vistos como explicações para o desempenho escolar. Por exemplo, atribui-se o fraco desempenho escolar de uma criança a um déficit em uma habilidade específica apontado em uma avaliação, ou essas instâncias internas são usadas para explicar o bom desempenho em alguma tarefa (Schrank \& Wendling, 2012). Por exemplo, "pessoas com uma inteligência fluida alta são capazes de descobrir soluções de problemas com bem pouca instrução" ou "pessoas com uma excelente capacidade de memória de curto prazo e bom controle da própria atenção parecem ter uma vantagem significativa em resolver problemas inéditos" (tradução livre de Schneider \& McGrew, 2013, p. 772). Assim, ainda hoje há a ideia de 
que a inteligência é uma característica interna e intrínseca aos indivíduos e que determina diferenças em desempenho acadêmico (e.g., Schneider \& McGrew, 2013).

Há também a difusão do chamado Fator de Inteligência Geral ou Fator $g$, que é visto como subjacente a todas as habilidades cognitivas e matematicamente derivado da saturação da variância compartilhada entre os subtestes de uma escala de inteligência. Charles Spearman (1863-1945) foi quem o mencionou pela primeira vez em seu artigo de 1904 (Wasserman \& Tulsky, 2005). Segundo Carroll (1997), o Fator $g$ é parcialmente herdado e considerado estável ao longo da vida, uma vez que é improvável que ele se altere, mesmo se outras habilidades melhorarem ("habilidades de leitura, ciências e matemática podem claramente ser melhoradas pela educação e por treino, mas não é tão claro assim que a inteligência geral possa ser treinada da mesma maneira", tradução livre de Carroll, 1997, p.35). Por causa disso, o fator $g$ é tido como um limite para a aprendizagem para os teóricos da área.

\section{CRÍTICAS FEITAS À PSICOMETRIA E AOS MÉTODOS DE ESTUDO DA INTELIGÊNCIA}

Dentro da perspectiva da Análise do Comportamento há críticas feitas tanto aos testes quanto às teorias da inteligência. Os testes avaliam uma entidade interna ao organismo e não os comportamentos testados em si. Isso gera uma explicação circular, em que a inteligência é apontada como a causa de um comportamento eficiente, ao mesmo tempo em que o comportamento é tomado como evidência de que a pessoa é inteligente (Schlinger, 2003). Tais entidades internas não podem ser manipuladas, pela falta de uma quantidade suficiente de informação sobre elas, isto é, não sabemos a quais variáveis ambientais elas estão funcionalmente relacionadas (Schlinger, 2003; Howe, 1988; Skinner, 1953).

Muitas das críticas direcionadas aos testes de inteligência e aos métodos usados para estudá-la são relacionadas ao uso indevido da estatística. A estatística é uma ferramenta útil para a ciência, contudo, a aplicação de alguns de seus parâmetros não é apropriada em alguns contextos. Por exemplo, a distribuição normal é adequada para expressar diferenças métricas em populações, mas não é pertinente para habilidades ou proficiência em algo. Uma distribuição de frequência normal é esperada quando a parte variável das medidas em questão pode ser expressa como a soma de muitas contribuições individualmente pequenas, mutuamente independentes e variáveis, o que não é o caso da inteligência (Layzer, 1972).

Como a inteligência é produto de um processo desenvolvimental complexo, as influências genéticas e ambientais são intrinsecamente misturadas. Assim, não há como fazer uma estimativa da proporção da capacidade cognitiva que é devida a fatores genéticos ou ambientais (Layzer, 1972). Hayes (1993) exemplifica como pode ser inapropriado tentarmos separar as influências genéticas das ambientais. Se a "fala metafórica" tivesse sido estudada e atribuída $80 \%$ a causas genéticas, imaginemos dois gêmeos idênticos geneticamente predispostos à fala metafórica. Agora, imaginemos que eles foram criados separadamente, sendo que um deles viveu em um ambiente extremamente empobrecido (em uma caixa de papelão, sendo alimentado por uma sonda). Seria muito provável que o gêmeo que viveu na caixa de papelão nem adquirisse linguagem. Portanto, a característica vista previamente como $80 \%$ genética se tornaria $100 \%$ ambiental.

A estabilidade do QI durante a vida é um artifício estatístico. O valor do QI torna-se arbitrário por conta da normatização dos escores dos testes. Com isso, mesmo com uma melhora no desempenho (a criança acertar cada vez mais questões do teste, por exemplo), o QI pode continuar o mesmo. Mais especificamente, o valor do QI serve apenas para comparar um indivíduo com relação ao seu próprio grupo, por exemplo, se ele tem um escore mediano, abaixo, ou acima da média. Por isso o teste tem que ser modificado e repadronizado para a aplicação em outro grupo, como é o caso da adaptação a um outro país. Por causa dessa limitação, aparece a demanda de uma medida de inteligência independente da população estudada, em que haja a descrição de diferenças de repertório em exposição a variáveis ou em taxa de mudança (Skinner, 1953; Schlinger, 2003). Com isso, poder-se-iam distinguir comportamentos que são mais relevantes em diferentes contextos (acadêmico, prático, ou emocional, por exemplo) ou subdividi-los e categorizá-los usando outros critérios socialmente relevantes (Schlinger, 2003).

O uso de critérios estatísticos para definir conceitos comportamentais também é alvo de severas críticas. Para estabelecer tais critérios, o foco é direcionado aos produtos comportamentais e não aos seus processos (i.e., as respostas do indivíduo e as variáveis do ambiente). Assim, com a criação de limites arbitrários (estatísticos) para a "normalidade", falhamos em identificar as fontes de variabilidade nas quais o critério se baseia, ou seja, há o critério por que há a ignorância do quê influencia aquele determinado comportamento (Sidman, 1986). Por exemplo, crianças com escores próximos no WISC-III podem ser classificadas diferentemente: a que apresenta escore mais baixo pode ser considerada com capacidade intelectual abaixo da média, enquanto que outra com o escore um pouco superior pode estar na faixa da normalidade. Poucos pontos de diferença podem determinar a adoção de medidas educacionais diferenciadas para cada uma das crianças. Entretanto, não sabemos se há uma diferença significativa no repertório delas. É provável que não haja e que a diferença de acertos seja muito pequena para justificar decisões educacionais distintas.

A amostra dos desempenhos a serem avaliados em um teste de inteligência também é questionada. Não há garantia de que habilidades amplas sejam testadas com as tarefas específicas apresentadas nos testes. Para que isso ocorresse, os testes teriam que avaliar os processos usando estímulos e respostas arbitrárias, para que o conhecimento prévio não influenciasse na avaliação (Sidman, 1986).

A concepção de que a capacidade cognitiva é majoritariamente hereditária, determinante do desempenho 
acadêmico e, portanto, um limite para a aprendizagem (Carroll, 1997), recebeu oposições na área. A literatura ressalta que limites biológicos não podem ser facilmente inferidos - limites não são descobertos pela presença de algo, mas na ausência. Isto é, quando uma criança com deficiência tem demonstrado dificuldades de aprendizagem de determinadas respostas (i.e., a ausência de modificação do comportamento), o professor dela pode atribuir isso a um limite biológico de aprendizagem. No entanto, o que se conhece sobre limites biológicos foi observado em ambientes conhecidos e ainda não se sabe o que poderia acontecer em outros ambientes que pudessem ser mais favoráveis à aprendizagem (Hayes, 1993; Roche \& Barnes, 1997).

Alguns estudos demonstraram o papel central do ambiente para o desenvolvimento intelectual infantil, tais como Hart e Risley (1995) e Smith, Eikeseth, Klevstrand, \& Lovaas (1997). Hart e Risley (1995) acompanharam longitudinalmente 42 famílias norte-americanas com crianças de um a dois anos, por dois anos e meio. Os autores tinham o intuito de identificar as experiências de crianças pequenas que se correlacionariam com sua taxa de vocabulário aos quatro anos de idade. A principal descoberta foi que as crianças que foram mais expostas à fala falavam mais, eram mais propensas a perguntar "por quê" e a querer saber como as coisas funcionavam. Mais experiência com proibições e retaliações foram associadas com um crescimento mais lento do vocabulário, um vocabulário menos variado e escores mais baixos na escala de inteligência Stanford-Binet. O crescimento e uso de vocabulário foram fortemente correlacionados com o QI. Um acompanhamento posterior das crianças mostrou que a quantidade de experiência com linguagem também é fortemente correlacionada com as habilidades delas aos três e aos nove anos. Neurologicamente, a infância é um período crítico para o desenvolvimento cortical, que depende da quantidade de estimulação do sistema nervoso, que, por sua vez, depende das experiências que a criança tem (Hart \& Risley, 1995).

Os resultados de Smith et al. (1997) corroboraram os dados de Hart e Risley. Smith et al. observaram um aumento significativo no QI de crianças que participaram de um programa intensivo de intervenção precoce (30 horas de intervenção ou mais por semana durante dois anos), mas eles não atingiram os mesmos resultados com crianças que tiveram menos intervenção (10 horas ou menos por semana pelo mesmo período). Portanto, novamente a diferença na exposição a oportunidades de aprendizagem demonstrou ser uma variável crítica, que pode determinar os futuros desempenhos das crianças.

\section{DESENVOLVENDO AS BASES PARA UM ESTUDO COMPORTAMENTAL DA INTELIGÊNCIA}

Skinner criticava a forma como características (traits) avaliadas em testes acabavam sendo tratadas como "entidades" causadoras de comportamentos, como no caso da inteligência. Para ele, ao dizermos que alguém é inteligente, estamos falando sobre diferenças em processos pelos quais os indivíduos passaram. Segundo ele, o que acontece no caso do indivíduo considerado inteligente é que ele supostamente demonstra condicionamento e extinção mais rápidos e, também, forma discriminações mais rapidamente. Para o caso de um indivíduo que obtém um alto escore em um teste de habilidades (achievement test), como um teste de inteligência, o resultado pode ser devido à exposição a certas variáveis, à velocidade em que essas variáveis fizeram efeito no organismo, ou ambas as situações (Skinner, 1953).

No entanto, o desenvolvimento de pesquisas em de Equivalência de Estímulos proporcionou analisar repertórios comportamentais relacionados ao que outras áreas da psicologia chamam de "inteligência". O paradigma de Equivalência de Estímulos permite compreender habilidades intelectuais em termos funcionais. Mais especificamente, o trabalho seminal de Murray Sidman (Sidman, 1971, 1994; Sidman \& Tailby, 1982) foi o marco inicial da possibilidade de se estudar a linguagem e processos cognitivos como relações complexas entre estímulos, sem que fosse necessário apoiar-se em processos internos ao organismo, inacessíveis a observadores (ver de Rose, de Souza, \& Hanna, 1996, sobre o estabelecimento de pré-requisitos de leitura, e Carpentier, Smeets, \& Barnes-Holmes, 2003, sobre o estabelecimento de raciocínio analógico).

A principal premissa da equivalência de estímulos é que, ensinadas algumas relações entre estímulos, outras emergem sem a necessidade de treino explícito. Dessa forma, estímulos nunca antes diretamente relacionados podem passar a se relacionar, e.g. se $A=B$ e $\mathrm{B}=\mathrm{C}$, então $\mathrm{A}=\mathrm{C}$ (Sidman, 1994). Essa propriedade gerativa da Teoria de Equivalência de Estímulos é chamada de responder relacional derivado no contexto da Teoria das Molduras Relacionais e será abordada mais adiante no texto.

Equivalência de estímulos é um conceito matemático também usado para descrever um fenômeno comportamental (Sidman, 2000). Mais especificamente, quando dois estímulos ou mais evocam a mesma resposta de uma pessoa, eles são ditos "funcionalmente equivalentes". Por exemplo, imagine que você está quase saindo de casa e ouça no rádio que a umidade do ar está baixa hoje. Você volta à geladeira e pega uma garrafinha de água. No trabalho, você lê uma manchete no jornal que menciona a baixa umidade do ar novamente e lembra de pegar sua garrafinha de água. Nos dois casos, tanto o estimulo auditivo, quanto o visual, evocam a mesma resposta de pegar a garrafinha de água (ver Hayes, Blackledge, \& Barnes-Holmes, 2001).

A possibilidade de realizar uma descrição comportamental de fenômenos tais como a maneira pela qual aprendemos o "significado" de uma palavra, ou então como objetos, palavras faladas e escritas tornam-se "substituíveis" entre si, mostrou-se muito promissora. Isso motivou muitas pesquisas e há um grande volume de publicações que tratam desse tópico (Dymond \& Roche, 2012; Hayes, Barnes-Holmes, \& Roche, 2001; Rehfeldt \& Barnes-Holmes, 2009; Sidman, 1994; Törneke, 2010).

A existência de outras formas de relações entre estímulos que vão além de relações de igualdade foi uma condição que favoreceu a complementação da teoria para 
que ela englobasse esses outros tipos de relações, tais como oposição, hierarquia, dentre outros (PérezAlmonacid, 2012). A Relational Frame Theory, ou Teoria das Molduras Relacionais (TMR), apareceu nesse contexto de demanda de ampliação dos tipos de relações abordados (Hayes et al., 2001).

Repertórios relacionais são aprendidos principalmente por condicionamento operante. As evidências de que a aprendizagem desses repertórios envolva condicionamento operante é que o comportamento operante é flexível e influenciado por suas condições antecedentes e por suas consequências, o que pode ser observado no responder relacional. Além disso, a aprendizagem operante se desenvolve de maneira gradual, $\mathrm{e}$ isso coincide com a forma como ocorre $\mathrm{o}$ desenvolvimento do responder relacional derivado em crianças (Törneke, 2010).

Uma moldura relacional é uma metáfora para um tipo de relação entre estímulos (e.g., similaridade, oposição, comparação, etc.) que pode ser aplicada a qualquer objeto ou evento. Molduras relacionais são consideradas "operantes puramente funcionais", isto é, eles descrevem o padrão de resposta visto em vários membros da moldura, que podem ser muito diferentes entre si, mas que são funcionalmente similares. Um exemplo seria uma menina ouvir a relação "mais que" em diferentes contextos, tais como: seu irmão diz que tem mais bolinhas de gude do que o amigo; sua colega de classe reclama que a professora emprestou mais lápis de cor a outros alunos em uma atividade em grupo; o dono do mercadinho diz que ela precisa de mais moedinhas do que ela tem para poder comprar um pirulito. Após ela ser exposta a muitas e diferentes instâncias de "mais que", o responder a qualquer estímulo por meio dessa relação de comparação se torna mais fluente ou generalizado, fazendo com que as formas de estímulos e respostas sejam menos relevantes para a aplicação apropriada da relação (Berens \& Hayes, 2007).

A TMR busca identificar padrões na maneira como ocorre a aprendizagem das relações entre estímulos, com a finalidade de prover informações sobre quais são as condições a que um indivíduo tem que ser exposto para aprender quaisquer tipos de relações. Por exemplo, o uso consistente de certos termos em certas configurações (tais como a presença da expressão "mais que"; o primeiro termo ser sempre "mais que" o segundo e isso implica que o segundo termo é "menos que" o primeiro) é o que nos fornece o "significado" dessas pistas (que geralmente são verbais). Pela perspectiva da TMR, há uma interdependência entre linguagem, repertórios relacionais e cognição, uma vez que todo evento "emoldurado relacionalmente" torna-se verbal (Stewart \& Roche, 2013). As molduras relacionais possuem três propriedades definidoras: implicação mútua, implicação combinatória e transformação de função (Hayes et al., 2001). Simplificando, a implicação mútua significa que, se qualquer estímulo se relaciona com outro, o segundo também se relaciona com ele. Implicação combinatória significa que: se $\mathrm{K} \mathrm{r}_{1} \mathrm{Z}$ e $\mathrm{Z} \mathrm{r}_{2} \mathrm{~N}$, então, $\mathrm{K} \mathrm{r}_{3} \mathrm{~N}$ - ou seja, dois ou mais estímulos nunca diretamente relacionados entre si passam a se relacionar por intermédio de outro. A transformação de função ocorre quando um dos estímulos que participa de uma rede relacional adquire uma nova função. De acordo com Hayes et al. (2001), o tipo de relação derivada entre estímulos determina como se dá a transformação de funções entre eles. O exemplo fornecido pelos autores é: se aprendido que A é o oposto de B, e A adquire funções punitivas, muito provavelmente $\mathrm{B}$ terá funções reforçadoras para o indivíduo (p. 32).

Uma moldura relacional seria aprendida pela exposição a múltiplos exemplares e essa aprendizagem ocorre naturalmente na interação da criança com seus cuidadores ou pares. Inicialmente, a moldura seria aprendida por meio de relações físicas ou não arbitrárias entre estímulos; somente depois ela se torna arbitrariamente aplicável (Hayes et al., 2001). Imagine uma outra situação em que uma criança ouça que uma mesa é maior do que uma cadeira, que seu pai é maior do que seu irmãozinho, que um prédio é maior do que uma casa e que uma melancia é maior do que uma maçã. Embora fisicamente diferentes, as situações apresentam semelhanças no tipo de relação guardada entre elas. Sendo assim, com a exposição a múltiplos exemplares, a criança identifica que o termo "maior" relaciona-se ao tamanho físico. Então, com o passar do tempo, consegue começar a aplicá-lo a situações arbitrárias como dizer que a moeda de dez centavos é duas vezes maior do que a de cinco embora a primeira seja fisicamente menor do que a segunda.

Quando o processo de aquisição das molduras relacionais é descrito, a criação de procedimentos para ensinar essas relações é viabilizada. Por exemplo, Y. Barnes-Holmes, D. Barnes-Holmes, Smeets, Strand, e Friman (2004) e Y. Barnes-Holmes, D. Barnes-Holmes, e Smeets (2004) treinaram com sucesso, respectivamente, um operante generalizado de mais/menos que e de oposição em crianças de quatro a seis anos. Berens e Hayes (2007) replicaram Y. Barnes-Holmes et al. (2004) com o intuito de investigar se as relações de comparação poderiam ser, de fato, tratadas como um operante relacional, mas realizaram um controle mais rigoroso de variáveis. No total, foram ensinadas relações comparativas de mais/menos que a quatro pré-escolares. Algumas evidências fornecem suporte para a hipótese das relações de comparação serem um operante relacional: no início do procedimento, nenhuma criança apresentava essas relações e ao final do experimento, elas haviam aprendido; além disso, algumas participantes apenas conseguiram ter sucesso nos treinos das relações arbitrárias quando foi dada a elas a oportunidade de treinar relações não arbitrárias de mais que e menos que - o que sugere que o responder relacional não arbitrário é um pré-requisito para a aquisição do responder relacional derivado, arbitrariamente aplicado. Assim, as molduras relacionais têm de ser encaradas como unidades psicológicas e não como unidades lógicas, uma vez que é necessária uma história de reforçamento para que elas se estabeleçam (Berens \& Hayes, 2007).

Hayes et al. (2001) descreveram nove molduras relacionais, sendo elas: 1) moldura relacional de 
coordenação, 2) de oposição, 3) de distinção, 4) de comparação, 5) de relações hierárquicas, 6) de relações temporais, 7) de relações espaciais, 8) de condicionalidade e causalidade e 9) de relações dêiticas. Os autores afirmaram que listaram apenas categorias gerais, não esgotando as possibilidades de molduras relacionais.

Um único estímulo pode participar de diversas molduras relacionais com uma infinidade de outros estímulos, formando redes relacionais (Hayes et al. 2001). Para esclarecer como isso se dá, pensemos em um estímulo qualquer - uma maçã, por exemplo. Apesar de haver diversos tipos de maçãs (grandes, pequenas, verdes, vermelhas, amareladas, frescas, ácidas, doces, etc.), todas elas participam de uma moldura relacional de coordenação com a palavra maçã (uma relação arbitrária). Além disso, ela participa de uma moldura relacional de hierarquia com o termo "fruta" e de uma moldura relacional de distinção com bananas.

Além disso, nas redes relacionais também podem existir relações entre as relações de estímulos, como por exemplo a analogia. Com o intuito de exemplificar o que é uma analogia, pode ser citado o seguinte exemplo: um elefante está para uma formiga, como um avião está para uma motocicleta. O caso está apresentado sob a notação $A: B:: C: D$. Os dois primeiros termos da analogia são animais e os outros dois são meios de transporte; contudo, em ambos os casos há uma relação de comparação de tamanho - o primeiro termo é bem maior do que o segundo, em cada um deles. Em outras palavras, apesar de os estímulos serem diferentes, o aspecto em comum é o tipo de relação existente entre cada par.

\section{O ESTUDO DA ANALOGIA PELA TEORIA DAS MOLDURAS RELACIONAIS}

$\mathrm{O}$ raciocínio analógico tem sido considerado um dos pilares principais da vida intelectual humana por diversos filósofos, médicos e psicólogos (Carpentier, Smeets, Barnes-Holmes, \& Stewart, 2004) e está relacionado à aquisição de novos conhecimentos em situações inéditas e ao entendimento de novos domínios ou áreas do conhecimento (Gentner, 1998; Richland, Morrison, \& Holyoak, 2006; Ruiz \& Luciano, 2011; Yanowitz, 2001). A capacidade de realizar analogias é a base de diversos outros processos cognitivos na idade adulta e também se relaciona à criatividade, à comunicação e à persuasão (Gentner, 1998). Além disso, é de fundamental importância na resolução de problemas (Gentner, 1998; Ruiz \& Luciano, 2011).

Skinner (1957) denominou a analogia de extensão genérica do tacto ${ }^{1}$. Para ele, a contingência de três termos explicaria a analogia, uma vez que uma propriedade em comum entre o estímulo original e o novo controlaria a resposta. A perspectiva Skinneriana de análise da analogia prevaleceu durante muitos anos. Por outro lado, podem ser apontadas algumas limitações desse enfoque, tais como a lacuna no estudo de analogias puramente relacionais (sem

\footnotetext{
${ }^{1}$ Segundo Skinner (1957), tacto é o operante verbal que está “em contato" com o ambiente, no sentido que o descreve. Ele é mantido por reforçamento generalizado (atenção).
}

elementos físicos em comum), a dificuldade da investigação experimental das condições em que surge esse repertório e, também, a dificuldade de identificação de quais variáveis favorecem o entendimento de algumas analogias (e metáforas) mais facilmente do que outras (Ruiz \& Luciano, 2012).

A TMR ofereceu uma definição de analogias, distinta daquela fornecida por Skinner (1957), que permitiu superar as antigas limitações ao seu estudo inclusive permitindo uma investigação experimental do fenômeno. Stewart, Barnes-Holmes, Hayes, e Lipkens (2001) definem relações de analogia como uma moldura relacional de coordenação entre relações treinadas ou derivadas, entre duas redes relacionais. A definição apresentada por eles baseia-se naquela apresentada na tese de doutorado de Regina Lipkens (1992), que foi o primeiro trabalho a considerar a analogia sob a perspectiva da TMR. No entanto, somente 17 anos depois foi publicado um artigo sobre a tese (Lipkens \& Hayes, 2009). Entre o período da conclusão da tese até a publicação do artigo, foram realizados outros estudos sobre analogia; contudo, foi utilizado o referencial da Equivalência de Estímulos, ao invés do da TMR. Assim, os artigos serão abordados na ordem cronológica de publicação, ou seja, primeiro aqueles que utilizaram a teoria da Equivalência, antes de relatar Lipkens e Hayes (2009).

Em linhas gerais, este é o procedimento mais utilizado nos experimentos de analogia que utilizaram a equivalência de estímulos: primeiro ensinavam-se relações arbitrárias entre estímulos abstratos; depois eram testadas as propriedades de simetria e transitividade (em alguns casos, essa etapa ocorre depois do teste de analogia: e.g., Experimento 2 de Barnes et al., 1997; Carpentier et al., 2003) e, por último, eram realizados os testes de equivalência-equivalência por meio de estímulos compostos. Nos testes de analogia (equivalênciaequivalência/não equivalência-não equivalência) os estímulos compostos poderiam ser formados por dois estímulos de uma mesma rede relacional, ou de redes relacionais distintas (por exemplo A1-B1 e A1-B2, respectivamente). A tarefa consistia em identificar o tipo de relação existente entre os estímulos que compunham o modelo e escolher aquele estímulo comparação formado por estímulos que apresentam o mesmo tipo de relação que o modelo, seja ela de equivalência ou de não equivalência - por exemplo, B2-C2 e A1-C1 e B2-C1 e A1-C3, respectivamente (Stewart \& Barnes-Holmes, 2004a, 2004b).

Barnes, Hegarty e Smeets (1997) publicaram o primeiro estudo sobre analogias sob a ótica da Análise Experimental do Comportamento. No entanto, como dito anteriormente, eles a interpretaram como relações de equivalência entre relações de equivalência entre estímulos. Barnes et al. (1997) realizaram alguns experimentos com o objetivo de investigar a possibilidade de estabelecimento de relação de equivalência entre relações de equivalência e, também, sobre a possibilidade de demonstrar a relação de equivalência entre relações antes de a equivalência entre estímulos ser testada. Foram ensinadas relações arbitrárias entre estímulos para crianças 
e adultos. Os autores obtiveram sucesso na tarefa e discutiram que o fato de uma criança de 12 e outra de nove anos terem demonstrado relações de equivalência entre relações de equivalência entre estímulos indica que esse tipo complexo de controle de estímulos prescinde de um longo treino educacional. Logo, os autores concluíram que esse tipo de relação não era subproduto da "lógica avançada" ou do "raciocínio matemático", como tradicionalmente eram vistos.

Alguns outros estudos que utilizaram o modelo de analogia baseado na Equivalência de Estímulos avaliaram a possibilidade de crianças pré-escolares aprenderem analogias, principalmente com relação a aspectos facilitadores e pré-requisitos envolvidos. As crianças de cinco anos do estudo de Carpentier, Smeets e BarnesHolmes (2002) falharam em desempenhar adequadamente nos testes de analogia, até que fosse dada a elas a oportunidade de realizar testes com as relações treinadas primeiro, ou seja, com aquelas que são pré-requisito para as mais complexas. Carpentier, Smeets, e Barnes-Holmes (2003) buscaram testar vários arranjos procedimentais para descobrir se crianças de cinco anos de idade teriam sucesso no teste de equivalência-equivalência, antes de realizarem o teste de equivalência; no entanto, todos falharam. Com isso, os autores concluíram algo um pouco diferente da conclusão do estudo realizado por Barnes et al. (1997): seus dados estavam de acordo com os da Psicologia do Desenvolvimento e os desempenhos das crianças, de fato, se relacionaram à idade e ao ensino acadêmico.

Voltando à perspectiva da TMR, e retomando o artigo de Lipkens e Hayes (2009), os autores realizaram quatro experimentos com estudantes universitários investigando dois pontos principais: (1) a possibilidade de estabelecerem-se analogias entre pares de estímulos de redes relacionais distintas, mesmo quando apenas as relações derivadas fossem análogas (2) utilizando diferentes topografias de resposta, tanto no treino, quanto nos testes (a seleção do estímulo correto, a produção do estímulo correto e a seleção da pista contextual correta). $\mathrm{O}$ uso de redes relacionais distintas tinha o objetivo de simular áreas distintas do conhecimento (por exemplo, realizar uma analogia entre algo da matemática com algo da biologia) e a topografia de resposta que produzia o estímulo correto buscava tornar a tarefa mais parecida com o que acontece naturalmente, quando fazemos uma analogia (por exemplo, em um contexto de resolução de problemas, geralmente criamos analogias que nos indicam como proceder e não selecionamos o que vamos fazer, por meio de analogias pré-fabricadas). De forma resumida, Lipkens e Hayes (2009) demonstraram que os participantes conseguiram identificar analogias entre redes relacionais diferentes. Isso ocorreu tanto com relações treinadas quanto derivadas e, também, com relações simétricas e não-simétricas. Além disso, os participantes foram capazes de obter sucesso em todos os tipos de topografia de resposta treinadas: quando deveriam selecionar o estímulo ou pista contextual corretos, ou quando tinham que produzir a resposta correta.
Alguns experimentos investigaram a possibilidade de demonstração de analogias que baseavam-se na abstração de propriedades formais de estímulos (Stewart, Barnes-Holmes, \& Smeets, 2001; Stewart, Barnes-Holmes, Roche, \& Smeets, 2002). Outros buscaram avaliar a validade do novo modelo de analogia por meio de dados neurofisiológicos (D. Barnes-Holmes et al., 2005) ou por meio da validade ecológica e externa comparação dos escores dos participantes nas tarefas relacionais, com os obtidos em testes padrão de analogia (Ruiz \& Luciano, 2011).

Considerando que analogias podem ser feitas entre diferentes áreas do conhecimento, Ruiz e Luciano (2011) desenvolveram um procedimento que permitiu o reconhecimento de relações analógicas entre domínios (redes relacionais). A resolução de um problema ou uma descoberta científica podem perpassar diferentes domínios do conhecimento e, por isso, os autores investigaram a possibilidade de se desenvolver um procedimento que permitisse o reconhecimento de relações analógicas entre duas redes relacionais diferentes e que assegurasse que isso acontecesse na primeira tentativa. Além disso, verificaram a possibilidade dos participantes reconhecerem as analogias entre as duas redes, sem que fosse necessário testar as implicações combinatórias de cada rede relacional. Para isso, os participantes aprenderam duas redes relacionais, cada uma composta de uma classe de equivalência entre três estímulos. Os testes consistiram no estabelecimento de relações combinatórias de similaridade e diferença entre as duas redes relacionais. Ruiz e Luciano (2011) obtiveram sucesso em ambos os objetivos com seus participantes (universitários).

Segundo alguns autores, a perspectiva da TMR contribui com o estudo da analogia por fornecer testes mais precisos do que os utilizados pela Psicologia Cognitiva ou pela Psicologia do Desenvolvimento para avaliar o raciocínio analógico (Stewart et al., 2002), principalmente por eles proverem um modelo de testes independente da utilização da linguagem natural (Lipkens \& Hayes, 2009). Um problema advindo do uso de testes padronizados de raciocínio analógico foi apontado por Carpentier et al. (2004): há a possibilidade de que o participante acerte a resposta sem ficar sob controle da analogia. Em um dos seus experimentos, dois dos termos da tarefa de analogia foram substituídos. Por exemplo, ao invés da tarefa ser apresentada em seu formato tradicional: aranha : teia $::$ abelha : ? (A : B :: C : D), os termos A e $\mathrm{C}$ foram substituídos pelas letras $\mathrm{X}$ e $\mathrm{Y}$. Assim, a tentativa ficava da seguinte forma: $X$ : teia $: \because Y$ : ?, seguida de quatro opções de resposta colméia/mel/formiga/mosca. A percentagem de acertos no teste foi de quase $100 \%$. Isso indica que os testes tradicionais necessitam ser modificados para não haver dúvidas de que as respostas dadas são baseadas em raciocínio analógico. Carpentier et al. (2004) ainda sugerem que os estudos de equivalênciaequivalência feitos anteriormente podem ter sofrido da mesma falha. Dessa forma, eles aconselharam modificações nas alternativas apresentadas nos testes de equivalência-equivalência para que seus resultados sejam totalmente atribuíveis ao raciocínio analógico. Segundo 
eles, o Experimento 4 descrito nesse mesmo artigo foi o primeiro que forneceu dados inequívocos sobre o modelo de estudo da analogia baseado na equivalência de estímulos.

\section{A RELAÇÃO ENTRE REPERTÓRIOS RELACIONAIS E INTELIGÊNCIA}

Como mencionado anteriormente, realizar analogias é apenas um dos tipos de formação de rede relacional que são relevantes sob o ponto de vista da cognição. Nesta seção, busca-se explorar as conexões entre repertórios relacionais e o funcionamento intelectual por meio dos achados de estudos da área da TMR. Desse modo, quando o termo "inteligência" é usado neste artigo dentro da perspectiva da TMR, ressalta-se que não estamos nos referindo a uma entidade mental inacessível que é vista como causa de comportamentos. Considera-se a "inteligência" como o repertório comportamental que engloba tipos distintos de responder relacional derivado. Assim, a "inteligência" é vista como a possibilidade de se estabelecerem redes relacionais de estímulos, sejam eles quaisquer relações e estímulos.

Apesar da interpretação relacional do raciocínio analógico já ter motivado muitos estudos, a primeira vez que a inteligência foi correlacionada ao desempenho em uma tarefa relacional foi com o estudo de O'Hora, Pelaez e Barnes-Holmes (2005). O’Hora et al. (2005) encontraram correlações entre o desempenho de participantes adultos em uma tarefa relacional complexa (que envolvia relações temporais e de similaridade/diferença) com a performance deles em três subtestes da Escala Wechsler de Inteligência (WAIS-III). $\mathrm{O}$ grupo de participantes que completou a tarefa relacional com sucesso $(N=31)$ obteve um escore significativamente maior nos subtestes de vocabulário e aritmética do que o grupo de indivíduos que falhou na tarefa $(N=44)$. Além disso, o número de tentativas corretas no treino também foi correlacionado com os escores dos subtestes de Aritmética e Vocabulário, mas não com o escore do Códigos (digit-symbol encoding). $\mathrm{O}$ escore do subteste de Aritmética e o de Vocabulário são utilizados para computar o QI Verbal. O subteste de Vocabulário ainda contribui com o índice de Compreensão Verbal. Tomadas em conjunto, essas evidências corroboram o fato de que repertórios relacionais e linguagem estão intimamente ligados.

Em um estudo posterior, O’Hora et al. (2008) decidiram aplicar todos os subtestes do WAIS-III e avaliar a relação deles com o desempenho de universitários $(N=81)$ em uma tarefa relacional temporal. O WAIS-III tem onze subtestes, sendo que sete deles fornecem o valor do QI verbal e seis o de QI execução. O WAIS-III também fornece o valor de quatro índices: o de Compreensão Verbal, Organização Perceptual, Memória de Trabalho e Velocidade de Processamento. O QI Verbal é derivado dos desempenhos nos subtestes de Informação, Semelhanças, Vocabulário (esses três formam o índice de Compreensão Verbal), Aritmética e Dígitos (os dois últimos formam o índice de Memória de Trabalho). Adicionalmente, o QI Execução envolve os subtestes de Cubos, Raciocínio
Matricial, Completar Figuras (esses fornecem o índice de Organização Perceptual), Códigos - dígitos e símbolos, Procurar Símbolos (o índice de Velocidade de Processamento é formado pelos dois), Arranjo de Figuras e Armar Objetos. O resultado obtido replicou em parte os achados da pesquisa anterior: o desempenho na tarefa relacional foi um bom preditor do índice de Compreensão Verbal. No entanto, contrário aos achados de O'Hora et al. (2005), não houve correlação do desempenho com o subteste de Aritmética. O índice de Organização Perceptual também correlacionou-se com o desempenho nas tarefas, o que foi inesperado. Os autores buscaram explicar essa correlação por meio da análise verbal pragmática. Análise verbal pragmática é definida como a ocorrência de responder relacional arbitrário (como as relações emergentes, no caso da equivalência de estímulos) sob o controle de relações não arbitrárias do mundo físico (como as relações temporais da tarefa que os participantes realizaram). Pode ser informalmente caracterizada como uma "habilidade de resolução de problemas" (Hayes, Gifford, Townsend, \& BarnesHolmes, 2001). No caso do subteste Cubos (Block Design), que apresentou uma alta correlação com o desempenho na tarefa, os participantes deveriam utilizar nove cubos para criar um desenho igual ao mostrado pelo avaliador. Isso também envolve a análise verbal pragmática, uma vez que os participantes têm que organizar os cubos modificando a posição inicial deles com o objetivo de torná-los iguais ao modelo. A análise verbal pragmática também está presente em outros subtestes que compõem o índice de Organização Perceptual e foram correlacionados com o desempenho da tarefa, tais como Completar Figuras (Picture Completion) e Raciocínio Matricial (Matrix Reasoning).

Outros dados interessantes relatados por O’Hora et al. (2008) foram que as maiores correlações foram encontradas entre o treino relacional e ambos, o QI Total e o QI Execução. Portanto, como era de se esperar, a correlação maior foi com o índice de Organização Perceptual (em que estão envolvidos alguns dos subtestes que compõem o QI Execução) do que com o índice de Compreensão Verbal. A tarefa relacional também correlacionou-se com todos os subtestes que compõem o índice de Compreensão Verbal e com o subteste de Procurar Símbolos (Symbol Search).

$\mathrm{O}$ fato do desempenho na tarefa relacional, que utilizava figuras geométricas relacionadas arbitrariamente e era livre de conteúdo, ter sido correlacionado fortemente com a maioria dos subtestes do WAIS-III, é um indicativo de que ambos abrangem alguns repertórios similares. Essa constatação apoia o fato de que repertórios relacionais estão envolvidos no que geralmente conhecemos como capacidades cognitivas. Tomados em conjunto, os dois estudos (O'Hora et al., 2005, 2008) encontraram correlações entre quase todos os subtestes e índices do WAIS-III, exceto com os índices de Velocidade de Processamento e o de Memória de Trabalho.

A relação entre o escore de um teste de inteligência e a propriedade comportamental de flexibilidade foi investigada por O'Toole e Barnes-Holmes 
(2009). Foi utilizado um teste abreviado de inteligência (K-BIT - the Kaufman Brief Intelligence Test) e o IRAP (the Implicit Relational Assessment Procedure). O IRAP é aplicado por um software desenvolvido originalmente para avaliar atitudes implícitas a assuntos controversos e que estão sujeitos a vieses de desejabilidade social. A latência de resposta é mensurada em tentativas consistentes e inconsistentes, ou seja, em algumas tentativas que estão de acordo com atitudes e relações estabelecidas como verdadeiras para o participante ("navio é similar a barco"), ou em outras que as contrariam ("navio não é similar a barco"), respectivamente. Como as relações inconsistentes não são usualmente praticadas, elas podem fornecer uma importante medida de flexibilidade relacional ou cognitiva. Consequentemente, a diferença entre as latências obtidas nas tentativas consistentes e inconsistentes também fornece uma medida de flexibilidade relacional, isto é, quanto menor a diferença entre os dois escores, maior a flexibilidade.

O K-BIT tem apenas dois subtestes, um de vocabulário e outro de matrizes. O subteste de vocabulário avalia habilidades verbais, conhecimento de palavras e formação de conceito verbal. Por outro lado, o de matrizes avalia habilidades não verbais e a habilidade de resolver problemas por meio de tarefas em que há a necessidade de perceber relações e de completar analogias. Na pesquisa de O'Toole e Barnes-Holmes (2009) o escore do K-BIT foi correlacionado com as latências de respostas nas tentativas consistentes e inconsistentes do IRAP, bem como com a diferença da latência entre os tipos de tentativas, que envolviam relações de similaridade/diferença e antes/depois. Os resultados obtidos foram indicativos de que as tentativas inconsistentes (das relações antes/depois e similaridade/diferença) e a diferença entre as latências das tentativas inconsistentes e consistentes foram as medidas correlacionadas significativamente com o QI. O fato de que tanto o desempenho nas tentativas inconsistentes quanto a diferença entre as latências foram correlacionadas com o QI indicam que o objetivo de um programa de ensino não deva ser somente a aprendizagem de relações corretas e fluentes, mas sim a construção de repertórios relacionais flexíveis.

Outros achados de O'Toole e Barnes-Holmes (2009) apontaram correlações específicas de cada tipo de moldura relacional com diferentes subtestes. As tentativas inconsistentes das relações de similaridade/diferença foram correlacionadas com o QI Total e com o escore do subteste verbal, enquanto que as tentativas inconsistentes das relações temporais se correlacionaram com o QI Total e o subteste de matrizes. No entanto, a diferença entre as latências das tentativas consistentes e inconsistentes em ambas as tarefas de similaridade/diferença e antes/depois foi correlacionada com o subteste verbal, implicando que a flexibilidade do responder relacional para essas relações havia sido relacionada com o domínio verbal.

Medidas de desempenho verbal também foram correlacionadas com o responder relacional em uma tarefa de tomada de perspectiva em Gore, Barnes-Holmes, e Murphy (2010). Os participantes de Gore et al. (2010) tinham deficiência intelectual e foram avaliados tanto com o WASI (Escala Wechsler Abreviada de Inteligência), quanto com a segunda edição do British Picture Vocabulary Scale (BPVS-II), uma medida de habilidade receptiva de linguagem e idade mental verbal. O escore total do WASI, os índices de performance e a de habilidade verbal dos participantes foram correlacionados com o desempenho na tarefa. Encontrou-se uma correlação positiva entre a idade mental verbal do BPVSII e as relações simples (que envolviam somente uma das instâncias EU/VOC $\hat{E}$, AQUI/ALI ou AGORA/DEPOIS ${ }^{2}$ ). $\mathrm{O}$ escore do QI execução foi correlacionado com os itens que revertiam as relações da tarefa de tomada de perspectiva $^{3}$. Gore et al. (2010) levantaram a hipótese de que habilidades espaciais e visuais utilizadas na resolução dos subtestes, por exemplo o de cubos, podem estar envolvidas com a manipulação de estímulos relacionais não formais, como é o caso dos itens revertidos da tarefa. Se esse for o caso, há a possibilidade de que estímulos formais ou físicos possam ser utilizados no desenvolvimento de tratamentos para melhorar o desempenho em tarefas de tomada de perspectiva.

Mais recentemente, outras evidências corroboraram a conexão entre inteligência e desempenho relacional. Dixon, Belisle, Whiting e Rowsey (2014) desenvolveram um sistema de treino relacional para crianças com deficiência chamado PEAK (sigla proveniente do inglês para promovendo a emergência de conhecimento avançado) que é composto por quatro módulos: treino direto, generalização, equivalência e transformação de função. Em um estudo posterior, o desempenho no treino do PEAK foi fortemente correlacionado com o QI de participantes com autismo (Dixon, Whiting, Rowsey, \& Belisle, 2014).

Anteriormente, a inteligência era somente vista como um objeto de avaliação; intervenções nunca tinham como objetivo o aumento no quociente intelectual (QI) em si mesmo. Professores e profissionais da área da educação primavam por ensinar novas habilidades aos seus aprendizes que, com sorte, implicariam em um aumento em seus escores em testes de inteligência. No entanto, a descoberta de que repertórios de responder relacional são correlacionados com os escores de testes de inteligência possibilita intervenções que tenham como objetivo melhorar o funcionamento cognitivo.

\section{O ENSINO DE REPERTÓRIOS RELACIONAIS E O IMPACTO NA INTELIGÊNCIA}

Cassidy, Roche e Hayes (2011) investigaram se um treino relacional poderia favorecer o aumento do escore em um teste de inteligência padrão em crianças. Foram realizados dois estudos e em cada um deles foram treinadas molduras relacionais de similaridade, oposição e comparação (mais que/menos que) por meio de múltiplos

\footnotetext{
${ }^{2}$ Um exemplo de item seria: "Eu tenho um tijolo vermelho e você um verde. Qual tijolo eu tenho? E qual tijolo você tem?". ${ }^{3}$ Exemplo de item revertido: "Eu tenho um tijolo vermelho e você um verde. Se eu fosse você e você fosse eu, qual tijolo eu teria? Qual tijolo você teria?".
} 
exemplares. Além das molduras relacionais já mencionadas, o primeiro experimento também estabeleceu relações de equivalência na parte inicial do treino.

No primeiro experimento foram estabelecidas duas classes de estímulos equivalentes com três estímulos (sílabas sem sentido) em cada uma, por meio de tentativas computadorizadas de discriminação condicional. Se as crianças realizassem os testes de relações derivadas (simetria e transitividade) com sucesso, elas prosseguiam com o procedimento. Os treinos das molduras relacionais ocorreram da seguinte forma: primeiramente estabeleciase a pista contextual e depois o treino de relações arbitrárias por múltiplos exemplares. O treino da pista contextual tinha o objetivo de estabelecer um significado para estímulos anteriormente sem sentido - !!!!! para SIMILARIDADE, $\% \% \% \% \%$ para OPOSIÇÃO, ***** para MENOS QUE e \$\$\$\$ para MAIS QUE. Cada tentativa apresentava uma pista contextual, que "sinalizava" qual seria a escolha correta no contexto da tentativa, i.e., se ele o participante deveria escolher a alternativa com o estímulo similar, oposto, com menos ou mais itens.

Para o treino das relações arbitrárias também utilizaram-se sílabas sem sentido. Duas sílabas situavamse na parte de cima da tela, uma no extremo direito e outra no extremo esquerdo. Elas eram separadas por uma das pistas contextuais e nos cantos inferiores da tela encontravam-se as palavras "sim" e "não". O participante era orientado a tratar os estímulos da parte de cima da tela como se fossem uma frase e selecionar uma das alternativas do canto inferior dela. Após $100 \%$ de acertos nas relações ensinadas, os participantes realizariam o teste das relações derivadas (implicações mútua e combinatória). Caso falhassem em demonstrar as relações derivadas, elas eram treinadas e um novo conjunto de estímulos seria usado na repetição do treino das relações arbitrárias.

É importante ressaltar a sequência dos treinos: primeiro foi realizado o treino da pista contextual de SIMILARIDADE, depois o treino das relações arbitrárias envolvendo a pista contextual de similaridade e depois o teste das relações derivadas. Em seguida, o mesmo processo foi repetido para a pista contextual de OPOSIÇÃO e depois, para as pistas contextuais de MAIS QUE e MENOS QUE, no entanto, o treino das duas últimas ocorreu simultaneamente.

No primeiro estudo, quatro crianças de desenvolvimento típico foram avaliadas por meio do WISC-III UK na linha de base, depois do treino em equivalência e ao final do experimento, quando todos os treinos haviam sido concluídos. Ao final do experimento, quando comparados aos indivíduos controle, os participantes tinham aumentado de forma significativa seus QIs, por volta de dois desvios-padrão. No segundo estudo, oito participantes de 11 a 12 anos com deficiência intelectual passaram pelas mesmas avaliações e treinos e também foi encontrado um aumento estatisticamente significativo para o QI do grupo, embora menor do que o que foi obtido no primeiro experimento. Segundo Cassidy et al. (2011), os dois estudos demonstraram o papel central que o responder relacional exerce na cognição humana. Tal descoberta pode servir para basear intervenções bem sucedidas para pessoas com deficiência intelectual e que possam auxiliar no funcionamento cognitivo geral desses indivíduos.

\section{CONCLUSÃO}

O diálogo entre a Psicometria e Análise do Comportamento é produtivo para ambas. A contribuição da Psicometria é enorme e muito relevante, uma vez que foram os psicometristas que tornaram o funcionamento intelectual humano um objeto de estudo e criaram avaliações que nos permitem realizar medidas que se correlacionam fortemente com desempenho acadêmico (Schroeders et al. 2010). Por outro lado, a descoberta da ligação entre repertórios relacionais e o funcionamento cognitivo inaugurou novas possibilidades de pesquisa, avaliação e intervenção.

Devido a essas novas possibilidades, pode-se dizer que a Análise do Comportamento retoma alguns dos objetivos iniciais de Binet. Podemos interpretar a "inteligência inata", que Binet gostaria de avaliar, como pré-requisitos para aprendizagens relacionais mais complexas. Ainda, a interpretação da inteligência como repertórios relacionais permite que se façam testes que avaliem a capacidade de relacionar estímulos (podemos dizer que é a "habilidade de raciocinar" sobre coisas) ao invés de conhecimentos específicos (e.g., RAI em Cassidy et al., 2011; PEAK em Dixon, Belisle, et al., 2014; e TARPA em Moran, Stewart, McElwee, \& Ming, 2010). Além disso, Binet buscava identificar, com o resultado de sua escala, alunos que precisassem de educação especial. Para aqueles que precisassem, ele propôs atividades que denominou "ortopedia mental", em que eles "aprenderiam a aprender" (Gould, 1991). O treino relacional, proposto para aumentar o escore fornecido pelos testes de inteligência (Cassidy et al., 2011), tem o mesmo papel da "ortopedia mental" de Binet, já que ele ensina relações que são pré-requisito para outros tipos de aprendizagens mais complexas.

O potencial de intervenção oferecido pela interpretação da inteligência em termos relacionais é animador. No entanto, mesmo com os importantes avanços descritos, ainda há muitos pontos para serem investigados. Estudos futuros são necessários para buscar esclarecer quais repertórios relacionais estão envolvidos em cada processo cognitivo avaliado nos testes de inteligência, ou em cada um dos construtos descritos em modelos de inteligência. Já foram feitas algumas proposições de molduras relacionais envolvidas nos subtestes do WISC e de outros testes menos conhecidos no Brasil (Cassidy, Roche, \& O'Hora, 2010). Recentemente, também foram realizadas interpretações preliminares dos repertórios envolvidos nas habilidades descritas pelo modelo de inteligência Cattell-Horn-Carroll (Rabelo, 2015). Avanços importantes poderiam ser proporcionados pela execução de um plano abrangente de pesquisa que buscasse testar experimentalmente a relação dos repertórios relacionais, identificados nos esforços interpretativos, com os construtos descritos pelos modelos e testes de inteligência. 
O conhecimento da literatura sobre a relação entre os repertórios relacionais e o funcionamento cognitivo ainda é bastante escasso e incipiente. Há a necessidade de que os componentes críticos presentes no treino (aqueles que proporcionam a melhora no funcionamento intelectual) sejam identificados, bem como relacionar as melhoras em diferentes tipos de repertórios com o tipo de moldura relacional utilizada em cada treino. Intervenções mais efetivas poderão ser planejadas quando conseguirmos ter uma visão mais panorâmica de qual é o papel de cada um dos diferentes tipos de responder relacional derivado na melhora do funcionamento cognitivo. Neste sentido, a união de forças entre a Análise do Comportamento e a Psicometria pode ajudar ambas a atingir seu objetivo em comum (ver Skinner, 1964 e Schneider \& McGrew, 2013): usar o conhecimento produzido para intervir em déficits e, com isso, melhorar a qualidade de vida das pessoas.

\section{REFERÊNCIAS}

Barnes-Holmes, D., Regan, D., Barnes-Holmes, Y., Commins, S., Walsh, D., Stewart, I., Smeets, P. M., Whelan, R., \& Dymond, S. (2005). Relating derived relations as a model of analogical reasoning: Reaction times and event related potentials. Journal of the Experimental Analysis of Behavior, 84(3), 435-451.

Barnes-Holmes, Y., Barnes-Holmes, D., Smeets, P. M., Strand, P., \& Friman, P. (2004). Establishing relational responding in accordance with more-than and less-than as generalized operant behavior in young children. International Journal of Psychology and Psychological Therapy, 4(3), 531-558.

Barnes-Holmes, Y., Barnes-Holmes, D., \& Smeets, P. M. (2004). Establishing relational responding in accordance with opposite as generalized operant behavior in young children. International Journal of Psychology and Psychological Therapy, 4(3), 559-586.

Barnes, D., Hegarty, N., \& Smeets, P. M. (1997). Relating equivalence relations to equivalence relations: A relational framing model of complex human functioning. The Analysis of Verbal Behavior, 14, $57-$ 83.

Berens, N. M., \& Hayes, S. C. (2007). Arbitrarily applicable comparative relations: Experimental evidence for a relational operant. Journal of Applied Behavior Analysis, 40(1), 45-71.

Carpentier, F., Smeets, P. M., \& Barnes-Holmes, D. (2002). Matching functionally same relations: Implications for equivalence-equivalence as a model of reasoning. The Psychological Record, 52, 351-370.

Carpentier, F., Smeets, P. M., \& Barnes-Holmes, D. (2003). Equivalence-equivalence as a model of analogy: Further analysis. The Psychological Record, 53, 349-371.

Carpentier, F., Smeets, P. M., Barnes-Holmes, D., \& Stewart, I. (2004). Matching derived functionally-same stimulus relations: Equivalence-equivalence and classical analogies. The Psychological Record, 54, 255-273.
Carroll, J. B. (1997). Psychometrics, intelligence and public perception. Intelligence, 24, 25-52.

Cassidy, S., Roche, B., \& O'Hora, D. (2010). Relational Frame Theory and human intelligence. European Journal of Behavior Analysis, 11(1), 37-51.

Cassidy, S., Roche, B., \& Hayes, S. C. (2011). A relational frame intervention to raise intelligence quotients: A pilot study. The Psychological Record, 61, 173-198.

Clark, L. A., \& Watson, D. (1995). Constructing validity: Basic issues in objective scale development. Psychological Assessment, 7(3), 309-319.

de Rose, J. C., de Souza, D. G., \& Hanna, E. S. (1996). Teaching reading and spelling: Exclusion and stimulus equivalence. Journal of Applied Behavior Analysis, 29, 451-469.

Dixon, M. R., Belisle, J., Whiting, S. W., \& Rowsey, K. E. (2014). Normative sample of the PEAK relational training system: Direct training module and subsequent comparisons to individuals with autism. Research in Autism Spectrum Disorders, 8, 1597-1606.

Dixon, M. R., Whiting, S. W., Rowsey, K., \& Belisle, J. (2014). Assessing the relationship between Intelligence and the PEAK Relational Training System. Research in Autism Spectrum Disorders, 8, 1208-1213.

Dymond, S., \& Roche, B. (2012). Advances in Relational Frame Theory: Research and application. Oakland, CA: Context Press.

Gentner, D. (1998). Analogy. In W. Bechtel \& G. Graham (Eds.). A companion to Cognitive Science (pp.107113). Oxford: Blackwell.

Gore, N. J., Barnes-Holmes, Y., \& Murphy, G. (2010). The relationship between intellectual functioning and relational perspective-taking. International Journal of Psychology and Psychological Therapy, 10, 1-17.

Gould, S. J. (1991). A falsa medida do homem (V. L. Siqueira, Trad.). São Paulo: Martins Fontes (Obra original publicada em 1981).

Hart, B., \& Risley, T. R. (1995). Meaningful differences in the everyday experiences of young American children. Baltimore: Paul Brookes.

Hayes, S. C. (1993). Nature-nurture: two-headed arrows and wrong-headed questions. In: S. C. Hayes, L. J. Hayes, H. W. Reese, \& T. R. Sarbin (Eds.). Varieties of scientific contextualism (pp.317-319). Reno, NV: Context Press.

Hayes, S. C., Barnes-Holmes, D., \& Roche, B. (2001). Relational Frame Theory: A post-Skinnerian account of human language and cognition. New York: Plenum.

Hayes, S. C., Blackledge, J. T., \& Barnes-Holmes, D. (2001). Language and cognition: Constructing an alternative approach within the behavioral tradition. In: S. C. Hayes, D. Barnes-Holmes, \& B. Roche (Eds.) Relational Frame Theory: A post-Skinnerian account of human language and cognition (pp. 3-20). New York: Plenum.

Hayes, S. C., Gifford, E. V., Townsend, R. C., \& BarnesHolmes, D. (2001). Thinking, problem-solving and pragmatic verbal analysis. In: S. C. Hayes, D. BarnesHolmes, \& B. Roche (Eds.) Relational Frame Theory: 
A post-Skinnerian account of human language and cognition (pp. 87-101). New York: Plenum.

Howe, M. J. A. (1988). Intelligence as an explanation. British Journal of Psychology, 79, 349-360.

Layzer, D. (1972). Science or superstition? (A physical scientist looks at the IQ controversy). Cognition, 1, 265-299.

Lipkens, R. (1992). Analogical reasoning as arbitrarily applicable relational responding. Tese de doutorado não publicada. Reno, NV: University of Nevada.

Lipkens, R., Hayes, S. C., \& Hayes, L. J. (1993). Longitudinal study of the development of derived relations in an infant. Journal of Experimental Child Psychology, 56, 201-239.

Lipkens, R., \& Hayes, S. C. (2009). Producing and recognizing analogical relations. Journal of the Experimental Analysis of Behavior, 91(1), 101-126.

McGrew, K. S. (2009). CHC theory and the human cognitive abilities project: Standing on the shoulder of the giants of psychometric research. Intelligence, 37, 110 .

Moran, L. Stewart, I., McElwee, J., \& Ming, S. (2010). The Training and Assessment of Relational Precursors and Abilities (TARPA): A preliminary analysis. Journal of Autism and Developmental Disorders, 40(9), 1149-1153.

O’Hora, D., Pelaez, M., \& Barnes-Holmes, D. (2005). Derived relational responding and performance on verbal subtests of the WAIS-III. The Psychological Record, 55, 155-175.

O’Hora, D., Pelaz, M., Barnes-Holmes, D., Rae, G., Robinson, K, \& Chaudhary, T. (2008). Temporal relations and intelligence: Correlating relational performance with performance on the WAIS-III. The Psychological Record, 58, 569-584.

O'Toole, C., \& Barnes-Holmes, D. (2009). Three chronometric indices of relational responding as predictors of performance on a brief intelligence test: The importance of relational flexibility. The Psychological Record, 59, 119-132.

Pérez-Almonacid, R. (2012). El análisis conductista del pensamiento humano. Acta Comportamentalia, 20 (1), 49-68.

Rabelo, L. Z. (2015). Uma análise comportamental da inteligência: propostas de intervenção e diálogos com a Psicometria. Tese de doutorado. São Carlos, SP.: Universidade Federal de São Carlos.

Rehfeldt, R. A., \& Barnes-Holmes, Y. (2009). Derived Relational Responding: Applications for learners with autism and other developmental disabilities. Oakland: Context Press.

Richland, L. E., Morrison, R.G., \& Holyoak, K. J. (2006). Children's development of analogical reasoning: Insights from scene analogy problems. Journal of Experimental Child Psychology, 94, 249-273.

Roche, B., \& Barnes, D. (1997). The behavior of organisms? The Psychological Record, 47, 597-618.

Ruiz, F. J., \& Luciano, C. (2011). Cross-domain analogies as relating derived relations among two separate relational networks. Journal of the Experimental Analysis of Behavior, 95(3), 369-385.

Ruiz, F. J., \& Luciano, C. (2012). Relacionar relaciones como modelo analítico-funcional de la analogía y la metáfora. Acta Comportamentalia, 20 (1), 5-31.

Schelini, P. W. (2006). Teoria das inteligências fluida e cristalizada: início e evolução. Estudos em Psicologia, 11, 323-332.

Schlinger, H. D. (2003). The myth of intelligence. The Psychological Record, 53, 15-32.

Schneider, W. J., \& McGrew, K. S. (2013). Individual differences in the ability to process information. In: B. J. Irby, G. Brown, R. Lara-Alecio, \& S. Jackson (Eds.) The handbook of educational theories (pp.767-782). Charlotte: Information Age Publishing.

Schrank, F. A., \& Wendling, B. J. (2012). The WoodcockJohnson-III Normative Update: tests of cognitive abilities and tests of achievements. In: D. P. Flanagan, \& P. L. Harrison (Eds.). Contemporary intellectual assessment: theories, tests and issues ( $3^{\text {rd }}$ edition) (pp.297-335). New York: The Guilford Press.

Schroeders, U., Wilhelm, O., \& Bucholtz, N. (2010). Reading, listening, and viewing comprehension in English as a foreign language: One or more constructs? Intelligence, 38, 562-573.

Sidman, M. (1971). Reading and visual-auditory equivalences. Journal of Speech, Language and Hearing Research, 14, 5-13.

Sidman, M. (1986). The measurement of behavioral development. In: N. A. Krasnegor, D. B. Gray \&, \& T. Thompson (Eds). Advances in behavioral pharmacology. Volume 5: Developmental behavioral pharmacology (pp.43-52). Hillsdale, NJ: Erlbaum.

Sidman, M. (1994). Equivalence relations and behavior: A research story. Boston: Authors Cooperative.

Sidman, M. (2000). Equivalence relations and the reinforcement contingency. Journal of the Experimental Analysis of Behavior, 74(1), 127-146.

Sidman, M., \& Tailby, (1982). Conditional discrimination vs. matching to sample: An expansion of the testing paradigm. Journal of the Experimental Analysis of Behavior, 37, 5-22.

Skinner, B. F. (1953). Science and human behavior. New York: The Macmillan Company.

Skinner, B. F. (1957). Verbal Behavior. New York: Appleton-Century-Crofts.

Skinner, B. F. (1964). New methods and new aims in teaching. New scientist, 122.

Smith, T., Eikeseth, S., Klevstrand, M., \& Lovaas, O. (1997). Intensive behavioral treatment for preschoolers with severe mental retardation and pervasive developmental disorder. American Journal on Mental Retardation, 102, 238-249.

Stewart, I., \& Barnes-Holmes, D. (2004a). A modern behaviour-analytic approach to analogical reasoning. The Irish Psychologist, 30(6), 117-124.

Stewart, I., \& Barnes-Holmes, D. (2004b). Relational Frame Theory and analogical reasoning: Empirical investigations. International Journal of Psychology and Psychological Therapy, 4(2), 241-262. 
Stewart, I., Barnes-Holmes, D., Hayes, S. C., \& Lipkens, R. (2001). Relations among relations: Analogies, metaphors, and stories. In: S. C. Hayes, D. BarnesHolmes, \& B. Roche (Eds.) Relational Frame Theory: A post-Skinnerian account of human language and cognition (pp. 73-86). New York: Plenum.

Stewart, I., Barnes-Holmes, D., Roche, B., \& Smeets, P. M. (2001). Generating derived relational networks via the abstraction of common physical properties: A possible model of analogical reasoning. The Psychological Record, 51, 381-408.

Stewart, I., Barnes-Holmes, D., Roche, B., \& Smeets, P. M. (2002). A functional-analytic model of analogy: Relational frame analysis. Journal of the Experimental Analysis of Behavior, 78(3), 375-396.

Stewart, I., \& Roche, B. (2013). Education, intellectual development, and Relational Frame Theory. In S. Dymond \& B. Roche (Eds.) Advances in Relational Frame Theory: research \& application (pp. 177197). Oakland: Context Press.

Törneke, N. (2010). Learning RFT: An introduction to Relational Frame Theory and its clinical application. Oakland: Context Press.

Wasserman, J. D., \& Tulsky, D. S. (2005). A history of intelligence assessment. In D. P. Flanagan, \& P. L. Harrison (Eds.), Contemporary intellectual assessment: Theories, tests, and issues (pp. 3-22). New York: The Guilford Press.

Yanowitz, K. L. (2001). Transfer of structure-related and arbitrary information in analogical reasoning. The Psychological Record, 51, 357-379.

Recebido em 28/08/2015

Aceite final em 06/12/2015

Editora: Deisy G. de Souza 